\title{
Neuromarketing: Tecnologías, Mercado y Retos
}

\author{
Sergio MONGE BENITO ${ }^{1}$ \\ Vanesa FERNÁNDEZ GUERRA² \\ Universidad del País Vasco / Euskal Herriko Unibertsitatea
}

\begin{abstract}
RESUMEN:
Los avances de las neurociencias en las últimas décadas han traído la oportunidad de utilizar sus tecnologías al servicio de la investigación en marketing. Existen numerosas controversias en torno a esta nueva aplicación de la tecnología, a la que se ha comenzado a llamar neuromarketing. Este artículo recoge un estado de la cuestión que analiza las diferentes tecnologías, los actores del mercado y los principales problemas y debates que están surgiendo en torno a esta disciplina.
\end{abstract}

PALABRAS CLAVE: neuromarketing; consumer science; EEG; fMRI; neurociencias; investigación de mercados

TITLE: Neuromarketing: Technologies, Market and Challenges

\begin{abstract}
:
The advances of neuroscience in the last decades have brought opportunities to use its technologies for market research. The application of these technologies, which has been called neuromarketing, has been controversial. This article analyzes current issues in this field of knowledge: different technologies, companies in this market and major challenges and discussions.
\end{abstract}

KEY WORDS: neuromarketing; consumer science; EEG; fMRI; neuroscience; market research.

\section{Introducción}

El objetivo del marketing debería ser alcanzar un equilibrio mutuamente beneficioso entre la oferta de los productores y los deseos o necesidades de los consumidores. Para obtener ese objetivo, los responsables de marketing utilizan todo tipo de técnicas de investigación que les ayudan a conocer qué está en la mente de sus consumidores. Algunos ejemplos de técnicas son los grupos de discusión (focus groups), las entrevistas en profundidad o las encuestas. Todas estas técnicas bien establecidas dependen de las declaraciones verbales de los entrevistados.

1 Sergio Monge es Doctor en Comunicación Audiovisual y Publicidad y Profesor Adjunto del Departamento de Comunicación Audiovisual y Publicidad de la UPV/EHU. Se le puede contactar en sergio.monge@ehu.es

2 Vanesa Fernández es Licenciada en Comunicación Audiovisual y Profesora del Departamento de Comunicación Audiovisual y Publicidad de la UPV/EHU. E-mail: vanesa.fernandez@ehu.es. 
Los enormes avances en las últimas tres décadas en el campo de las neurociencias, de la mano de científicos como Joseph Ledoux (New York University), Daniel Kahneman (Princeton University), Muhzarin Banaji (Harvard University), Daniel Schacter (Harvard University), Antonio Damasio (University of South California), John Bargh (Yale University) o Robert Zajonc (Stanford University), nos han permitido visualizar a un consumidor muy diferente del que nos venían dibujando los modelos económicos anteriores. Las claves para entender a este nuevo consumidor son:

1. El homo aeconomicus actúa de manera irracional. La mayoría de los modelos económicos se basaban en una visión romántica del ser humano en la que éste tomaba las decisiones más favorables racionalmente. La realidad del mercado nos muestra que habitualmente actuamos de manera irracional.

2. Para comprender lo que pasa en la mente de los consumidores, es necesario ir más allá de las declaraciones verbales. Las declaraciones verbales, incluso en el caso en el que no pretenda engañarnos, no son testimonios completamente fiables porque pueden existir factores inconscientes para el sujeto.

3. Existe todo un universo de decisiones inconscientes. Algunas decisiones las tomamos en base a información que poseemos pero de la que no somos conscientes: se producen en un segundo plano de nuestra consciencia.

Cuando utilizamos técnicas basadas en declaraciones verbales para investigar determinados temas especialmente sensibles (política, sexo...), el consumidor puede verse tentado a mentir o a responder utilizando el estereotipo de lo correcto. Incluso aunque el consumidor esté dispuesto a decirnos la verdad en sus declaraciones verbales, puede que no conozca las respuestas que pretendemos obtener. De acuerdo a la mayoría de las estimaciones, el $95 \%$ de los pensamientos, las emociones y el aprendizaje se producen a nivel inconsciente ${ }^{3}$.

Ante esa necesidad de explorar el inconsciente de los consumidores para obtener respuestas más fiables sobre su comportamiento y percepciones, autores como Zaltman han desarrollado y aplicado técnicas de análisis del pensamiento metafórico explicitado mediante imágenes en entrevistas en profundidad. La «Técnica de Elicitación de Metáforas de Zaltman» (ZMET) es el resultado de sus investigaciones y ha sido aplicada a diferentes temas (Coutler, Zaltman, Coutler, 2001; Zaltman, Coutler, 1995) para revelar los pensamientos profundos que los consumidores.

Para abordar el mismo problema desde otra óptica, científicos y profesionales (incluyendo al propio Zaltman) están intentando utilizar técnicas de las neurociencias con el mismo objetivo: explorar el inconsciente de los consumidores. El neu-

3 Zaltman, G. (2003): How Customers Think. Boston (MA), Harvard Business School Press, 40. 
romarketing consiste en la aplicación de las técnicas de las neurociencias a la investigación de marketing tradicional. A través de tecnologías como la Electroencefalografía (EEG) o la Resonancia Magnética Funcional (fMRI), las «respuestas» de los entrevistados a distintos estímulos (por ejemplo, anuncios publicitarios) son «leídas» directamente de su actividad cerebral. Las neurociencias permiten, por ejemplo, averiguar qué nivel de atención está prestando los sujetos estudiados a un anuncio segundo por segundo y plano por plano. La promesa del neuromarketing es ir mucho más allá de ese punto para determinar preferencias (emociones positivas y negativas), probabilidades de recordar el estímulo o, en el mejor de los sueños de los comunicadores, «recetas persuasivas» infalibles dirigidas a nuestra mente inconsciente. Pero, de momento, todas esas promesas no son sino expectativas y lo que podemos encontrar en el neuromarketing actual es una metodología de investigación de mercados alternativa (muchas veces complementaria) a las técnicas convencionales.

A medida que este tema se ha tratado en libros de divulgación (Lindstrom, 2008; Braidot, 2009), noticias o artículos, se ha generado una enorme interés en torno al neuromarketing, tanto por parte de los profesionales de la comunicación y el marketing como de los ciudadanos en general. Los primeros ven una oportunidad sin precedentes para mejorar su arsenal persuasivo. Los segundos una amenaza similar a la que desató en los medios James Vicary cuando publicó los resultados de su famoso experimento de 1957 sobre publicidad subliminal (Crandall, 2006). Sin embargo, todo ese interés no ha propiciado una mejor comprensión de estas técnicas de investigación sino bastante desconcierto y desinformación en torno a sus posibilidades y aplicaciones reales.

Por este motivo, existe una preocupación en los ámbitos científicos, especialmente los más cercanos a las neurociencias y la neurobiología, que va creciendo a medida que los medios de comunicación tocan este tema con frivolidad o los profesionales de la comunicación se permiten utilizar simplificaciones (muchas veces completamente erróneas) de conceptos neurocientíficos para explicar fenómenos del mercado. No es de extrañar que todo tipo de profesionales se muestren deseosos de utilizar «neuropalabrería» en sus discursos, ya que esta se ha demostrado científicamente más convincente que las explicaciones convencionales (Weisberg, Keil, et al., 2008).

Ante la ausencia de un artículo académico en español que ofrezca un completo estado de la cuestión sobre el neuromarketing (tecnologías, mercados y retos), el presente artículo pretende servir de punto de partida para otros investigadores que pretendan adentrarse en este campo. La información que contiene se basa en las referencias bibliográficas que están convenientemente citadas (se ha hecho un especial esfuerzo en este punto), observación participante del principal foro internacional $^{4}$ que reúne profesionales de este ámbito y en entrevistas informales con pro-

4 Grupo «Neuromarketing» en la red profesional LinkedIn:

http://www.linkedin.com/groups?gid=852427 
fesionales y científicos en varios congresos o eventos sectoriales (entre los que se incluye Neuro Connections, 5-7 de febrero de 2009 en Cracovia, una de las mayores citas europeas sobre neuromarketing).

\section{Principales tecnologías de neuromarketing}

Las consultoras de este sector utilizan varias tecnologías propias de las neurociencias para realizar sus investigaciones. Las dos más populares son la electroencefalografía (EEG) y la Resonancia Magnética Funcional. A menudo combinan esas tecnologías neurocientíficas con otras mediciones biométricas, como puedan ser la medición de la respuesta galvánica de la piel, seguimiento ocular (eyetracking), medición del ritmo cardiaco o la electromiografía.

A continuación figura una descripción de las tecnologías más relevantes en este sector:

\subsection{Resonancia Magnética Funcional (fMRI)}

La Resonancia Magnética Funcional o fMRI (Wikipedia, 2011b) es una técnica que permite obtener imágenes del cerebro mientras éste realiza una tarea. La tecnología funciona midiendo, mediante magnetismo, cambios en los niveles de oxigenación de la sangre, que a su vez se relacionan con la actividad cerebral.

La fMRI no requiere inyección de sustancia alguna (no es invasiva) pero requiere que el sujeto se coloque en una máquina en forma de tubo que puede generar ansiedad claustrofóbica según el sujeto. La fMRI ofrece una excelente resolución espacial, ya que identifica perfectamente (1-10 mm de resolución) la zona del cerebro con mayor actividad. No obstante, la resolución temporal no es tan buena (110 s.) como la de otras tecnologías de neurociencias. En general, cuanto mayor es la resolución espacial, peor es la resolución temporal (necesita más tiempo para obtener cada imagen).

El uso de la fMRI es necesario para obtener información sobre la actividad de las partes más internas del cerebro, como por ejemplo el Nucleus Acumbens, que se ha vinculado a la intención de compra; la Ínsula, que se relaciona con la intensidad que tiene el gusto/disgusto por lo que se está viendo; el Cortex Prefontal Ventromedial (VMPFC), que parece estar implicado en procesos posteriores a las decisiones de marca; o el Córtex Cingulado Anterior (ACC), que permite medir el grado de contradicción percibida entre estímulos. Existe abundante literatura científica que atestigua la importancia de estas y otras regiones subcorticales para determinar la preferencia de los consumidores (Knutson, Adams et al., 2001; Izuma, Saito et al., 2001; Passmann, O’Doherty et al., 2001; Knutson, Rick, et al., 2007; Deppe, Schwindt, et al., 2007; Grosenic, Greer, et al., 2008; McClure, Li et al, 2004; Hare, O'Doherty, et al., 2008; Santos, Seixas, et al, 2011). 
Las máquinas de fMRI cuestan aproximadamente 1 millón de dólares americanos por Tesla y unos costes anuales de operación entre 100.000 y $300.000 \$$, lo que las sitúa fuera del alcance de la mayor parte de los investigadores comerciales 5 . Aunque se pueden encontrar bastantes estudios basados en fMRI en artículos académicos, la razón económica es la principal por la que, a pesar de su interés para el neuromarketing, no son la tecnología más utilizada por las consultoras.

\subsection{Electroencefalografia}

La electroencefalografía o EEG (Wikipedia, 2011a) es una de las técnicas más empleadas por las consultoras de neuromarketing por su reducido coste frente a la fMRI.

La actividad coordinada de miles de neuronas produce diferencias de potencial en el cuero cabelludo que pueden ser registradas utilizando electrodos en conjunción con amplificadores de señal. Es decir, colocando una serie de electrodos repartidos por la cabeza podemos hacernos una idea de en qué zonas de nuestro cerebro se está produciendo mayor actividad.

La EEG que toma datos del cuero cabelludo es una técnica no invasiva y silenciosa que es sensible a la actividad neuronal. Su resolución temporal la determina el hardware específico, pero típicamente mide el voltaje cada entre 1 y 3 milisegundos, lo que supone una excelente resolución temporal que permite incluso detectar fenómenos neurales muy breves que pasarían desapercibidos a la fMRI.

Sin embargo, la EEG tiene una resolución espacial muy limitada (según el número de electrodos, desde dos a varios cientos) y no ofrece datos de las partes más internas del cerebro, ya que la medición se realiza a nivel del cuero cabelludo.

Existe una cierta controversia con respecto a la tecnología que se debe utilizar en los electrodos de la EEG. Mientras que algunas consultoras (Sands Research) defienden el estándar académico de utilizar al menos 32 o 64 electrodos húmedos (con gel) para recoger la información, varias de las consultoras más importantes (EmSense y Neurofocus) han desarrollado tecnologías propias (EmBand ${ }^{6}$ Mynd $^{7}$, respectivamente) que utilizan electrodos secos más proclives a las interferencias de la actividad muscular.

La principal ventaja de la EEG frente a la fMRI es el coste, puesto que se pueden conseguir equipos por menos de 10.000 dólares $\mathrm{USA}^{8}$. Otra gran ventaja es que el EEG ofrece libertad de movimientos al sujeto, ya que éste puede moverse en una estancia (o una tienda) e interactuar (cosa que no podría hacer dentro de una fMRI, por ejemplo).

\footnotetext{
5 ARIEL, D.; BERNS, G.S. (2010): «Neuromarketing: the hope and hype of neuroimaging in business». Nature Reviews Neuroscience, April 2010, 11, 288.

http://www.emsense.com/technology.php

http://www.neurofocus.com/pdfs/Mynd_NeuroFocus.pdf

ARIEL, BERNS (2010), op. cit. 288.
} 
Una técnica común con esta tecnología es medir la asimetría de actividad entre las regiones izquierda y derecha del córtex frontal (Davidson, Ekman et al., 1990). La literatura científica al respecto sugiere que una actividad superior en la región izquierda se asocia bien con estados emocionales positivos, bien con la intención de acercarse a un objeto (Harmon-Jones, 2003). En algunos casos, se ha utilizado esta técnica para medir respuestas momentáneas a spots televisivos (Ohme, Reykowska et al., 2009; Ohme, Reykowska et al., 2010) aunque hay cierta controversia sobre si es necesario hacer mediciones más largas, de hasta 60 s., para obtener resultados aplicables con esta técnica (Hustler, Stevens et al., 2008).

\subsection{Magnetoencefalografía}

La actividad coordinada de las neuronas produce campos magnéticos además de las corrientes eléctricas que medía el EEG. La intensidad de estos campos es realmente pequeña pero puede ser medida por una técnica denominada magnetoencefalografía o MEG (Wikipedia, 2011c).

La EEG y la MEG son técnicas conceptualmente similares, pero la MEG ofrece una calidad de señal superior y una resolución temporal muy alta. Sin embargo, sus costes son muy superiores: un equipo completo incluyendo una habitación aislada magnéticamente puede costar unos 2 millones de dólares ${ }^{9}$. Por ello, no es una técnica tan popular como el EEG entre las consultoras de neuromarketing.

\subsection{Otras mediciones biométricas}

Las siguientes mediciones biométricas no son tecnologías de las neurociencias propiamente dichas, por lo que no se deberían encuadrar dentro del campo que estamos estudiando. Algunos incluso las han denominado «bodymarketing» en oposición al término «neuromarketing». No obstante, lo que sí es cierto es que las consultoras las emplean frecuentemente en sus estudios para complementar las mediciones «neuro». Éstas son algunas de las más habituales:

Electromiografía. La electromiografía o EMG es una técnica médica que consiste en la aplicación de pequeños electrodos de bajo voltaje en forma de agujas en el territorio muscular que se desea estudiar para medir la respuesta y la conectividad entre los diferentes electrodos. La EMG mide actividad eléctrica generada por los músculos, y las consultoras de neuromarketing lo emplean sobre todo para monitorizar los músculos faciales asociados con las respuestas emocionales involuntarias. Cuando somos sometidos a un estímulo (por ejemplo, un anuncio de televisión), los músculos de nuestra cara se mueven involuntariamente como reacción a lo que estamos viendo. Algunas de esas expresiones son de muy corta duración y difíciles de detectar a simple vista. La electromiografía (EMG) se utiliza como

$9 \quad$ Ibidem, 288. 
indicador de la valencia positiva o negativa de la reacción a los estímulos (es decir, gusto o disgusto), especialmente para estímulos visuales, auditivos, olfativos y gustativos.

FACS. No es una tecnología en sí, pero, en algunos casos, la observación de las expresiones faciales se realiza grabando la cara del sujeto y realizando una codificación manual de los músculos que se activan durante la exposición al estímulo. El sistema de codificación facial (FACS, Facial Action Coding System) más conocido y utilizado fue desarrollado por Paul Ekman en los setenta (Ekman, Friesen, 1978). En la actualidad, existe software informático que permite la codificación automática de las expresiones faciales (Azcarate, Hageloh et al., 2005; Salah, Sebe et al., 2009) y algunas compañías ${ }^{10}$ comercializan dichos paquetes de software para su uso en investigación de mercados.

Seguimiento ocular. Los sistemas de seguimiento ocular (eye-tracking) se emplean a veces junto con tecnologías de neuromarketing para identificar hacia dónde están mirando los sujetos. Cuando analizamos estímulos simples, como una imagen o una pieza audiovisual, resulta factible utilizar directamente tecnologías como el EEG, puesto que el sujeto le presta total atención al estímulo. Sin embargo, si pretendemos analizar el comportamiento del comprador en una tienda llena de estímulos (productos), necesitamos combinar el EEG con seguimiento ocular para saber exactamente qué está produciendo las reacciones cerebrales. La tecnología de seguimiento ocular ofrece información de contexto muy valiosa para combinar con los resultados de la tecnología de neuromarketing. Algunas empresas (iMotions, www.imotionsglobal.com) incluso están intentando utilizar métricas como la dilatación de las pupilas y la frecuencia de parpadeo para deducir no solo hacia dónde estamos mirando sino las emociones que estamos sintiendo al hacerlo en cada momento. La mayor parte de las consultoras, sin embargo, combinan el seguimiento ocular con otras técnicas para obtener esa misma información.

Respuesta galvánica de la piel. La actividad electrodérmica (EDA) es otro tipo de medición biométrica que se utiliza habitualmente por parte de las consultoras de neuromarketing. Es la misma tecnología descubierta por Feré (1881) que utiliza el polígrafo y se basa en la premisa de que la resistencia de la piel a la electricidad varía con la activación (arousal) que experimenta el sujeto. El arousal simpático genera una activación mayor de las glándulas sudoríparas, que a su vez provocan una mayor actividad electrodérmica (EDA) por la humedad resultante, lo que acaba alterando las propiedades bioeléctricas de la piel (Boucsein, 1992). Un estudio descubrió, estudiando tanto la EDA como el ritmo cardiaco, que los compradores y no compradores que se pasean por las tiendas de un centro comercial tienen distintos patrones de activación (Groeppel-Klein, 2005). La activación es una métrica

10 Affectiva, www.affectiva.com; Thirdsight, www.thirdsight.co; Noldus, www.noldus.com; nViso, www.nviso.ch 
que las consultoras de neuromarketing incorporan habitualmente a sus metodologías para detectar el impulso de compra en los sujetos estudiados.

Otras biométricas que pueden ser monitorizadas incluyen el ritmo cardiaco, la presión sanguínea, la respiración o la sudoración.

\section{El mercado internacional del neuromarketing}

El mercado internacional de empresas que realizan estudios con técnicas de neuromarketing es aún muy pequeño, con unos pocos actores con alcance internacional (la mayoría en EE.UU.). La reciente inversión de Nielsen en Neurofocus (The Nielsen Company, 2008) ha incrementado el interés de los inversores por este sector de la investigación de mercados. Otras empresas como EmSense han recibido también cuantiosas rondas de inversión (Reuters, 2009).

La siguiente tabla resume información sobre los principales proveedores globales de servicios de neuromarketing (empresa, web, sede, algunos científicos notables, tecnologías que emplea y miscelánea de notas). Algunos de ellos no utilizan tecnologías que proceden propiamente de las neurociencias. Lo que tienen en común es que todos ellos tratan de investigar la parte inconsciente de los procesos cerebrales y las implicaciones para el marketing desde las aportaciones de la tecnología:

\begin{tabular}{|c|c|c|c|c|}
\hline EMPRESA & SEDE & CIENTÍFICOS & TECNOLOGÍAS & NotaS \\
\hline $\begin{array}{l}\text { Neurofocus } \\
\text { neurofocus.com }\end{array}$ & $\begin{array}{l}\text { Berkeley, } \\
\text { California, } \\
\text { ESTADOS } \\
\text { UNIDOS }\end{array}$ & $\begin{array}{l}\text { Dr. A.K. } \\
\text { Pradeep, Dr. } \\
\text { Robert T. } \\
\text { Knight }\end{array}$ & $\begin{array}{l}\text { EEG, EDA, eye- } \\
\text { tracking, FACS. }\end{array}$ & $\begin{array}{l}\text { Neurofocus es una subsidiaria } \\
\text { de Nielsen. No participan de la } \\
\text { iniciativa de estandarización de } \\
\text { la ARF. }\end{array}$ \\
\hline $\begin{array}{l}\text { Sands Research } \\
\text { www.sandsresearch.com }\end{array}$ & $\begin{array}{l}\text { El Paso, } \\
\text { Texas, ES- } \\
\text { TADOS } \\
\text { UNIDOS }\end{array}$ & $\begin{array}{l}\text { Dr. Stephen } \\
\text { Sands }\end{array}$ & $\begin{array}{l}\text { EEG, eye- } \\
\text { tracking }\end{array}$ & $\begin{array}{l}\text { Integración vertical: desarro- } \\
\text { llan el hardware y software } \\
\text { para utilizar con el EEG. } \\
\text { Sistema de medición NES } \\
\text { (Neuro Engagement Score) }\end{array}$ \\
\hline $\begin{array}{l}\text { EmSense } \\
\text { www.emsense.com }\end{array}$ & $\begin{array}{l}\text { San Francis- } \\
\text { co, California, } \\
\text { ESTADOS } \\
\text { UNIDOS }\end{array}$ & $\begin{array}{l}\text { Dr. Antoine } \\
\text { Bechara, Dr. } \\
\text { Michael } \\
\text { Gazzaniga, } \\
\text { Dr. Read } \\
\text { Montague, } \\
\text { Dr.Thomas } \\
\text { Ramsoy, Dr. } \\
\text { Cynthia } \\
\text { Breazeal }\end{array}$ & $\mathrm{EEG}^{11}$, otras. & $\begin{array}{l}\text { Hardware propietario (Em- } \\
\text { Band y EmBand 24). Tiene } \\
\text { una base de datos con registros } \\
\text { de más de } 100.000 \text { sujetos. } \\
\text { Declaran realizar «neuromar- } \\
\text { keting cuantitativo» porque } \\
\text { utilizan muestras mayores que } \\
\text { lo habitual en el sector. No } \\
\text { participan de la iniciativa de } \\
\text { estandarización de la ARF. }\end{array}$ \\
\hline
\end{tabular}

11 El EEG utilizado por EmSense (EmBand y EmBand 24) se diferencia de otros del sector. Utilizan dos electrodos estratégicamente colocados en lugar de un gorro completo de 32 o 64 . Además, en lugar de ser electrodos húmedos, utilizan electrodos secos, lo que ha generado cierta controversia entre los académi$\cos$. 


\begin{tabular}{|c|c|c|c|c|}
\hline EMPRESA & SEDE & CIENTÍFICOS & TECNOLOGÍAS & NotaS \\
\hline $\begin{array}{l}\text { NeuroCompass } \\
\text { neurocompass.com }\end{array}$ & $\begin{array}{l}\text { Palo Alto, } \\
\text { California, } \\
\text { ESTADOS } \\
\text { UNIDOS }\end{array}$ & & fMRI & \\
\hline $\begin{array}{l}\text { Innerscope } \\
\text { www.innerscope.com }\end{array}$ & $\begin{array}{l}\text { Boston, } \\
\text { Massachusset, } \\
\text { ESTADOS } \\
\text { UNIDOS }\end{array}$ & $\begin{array}{l}\text { Dr. Carl } \\
\text { Marci }\end{array}$ & $\begin{array}{l}\text { EDA, eye- } \\
\text { tracking, otras. }\end{array}$ & $\begin{array}{l}\text { Combinación de hardware y } \\
\text { software propietario para } \\
\text { medir el engangement. No } \\
\text { utiliza técnicas de la neuro- } \\
\text { ciencia propiamente dicha. }\end{array}$ \\
\hline $\begin{array}{l}\text { Sensory Logic, } \\
\text { www.sensorylogic.com }\end{array}$ & $\begin{array}{l}\text { Minneapolis, } \\
\text { Minnesota, } \\
\text { ESTADOS } \\
\text { UNIDOS }\end{array}$ & Dr. Dan Hill & $\begin{array}{l}\text { FACS, eye- } \\
\text { tracking }\end{array}$ & $\begin{array}{l}\text { Investiga respuesta emocional } \\
\text { mediante codificación facial, } \\
\text { no neuromarketing }\end{array}$ \\
\hline $\begin{array}{l}\text { Buyology, Inc, } \\
\text { www.buyologyinc.com }\end{array}$ & $\begin{array}{l}\text { New York, } \\
\text { ESTADOS } \\
\text { UNIDOS }\end{array}$ & $\begin{array}{l}\text { (Martin } \\
\text { Linstrom) }\end{array}$ & Varía. & $\begin{array}{l}\text { Consultora de marca que } \\
\text { ofrece distintos servicios } \\
\text { relacionados con neuromarke- } \\
\text { ting a través de sus socios. }\end{array}$ \\
\hline $\begin{array}{l}\text { Gallup \& Robinson } \\
\text { www.gallup- } \\
\text { robinson.com }\end{array}$ & $\begin{array}{l}\text { Pennington, } \\
\text { New Jersey, } \\
\text { ESTADOS } \\
\text { UNIDOS }\end{array}$ & & Electromiografía & $\begin{array}{l}\text { Realiza también copytesting } \\
\text { tradicional (online y en perso- } \\
\text { na). Su sistema se denomina } \\
\text { CERA (Continuous Emotional } \\
\text { Response Analysis ) }\end{array}$ \\
\hline $\begin{array}{l}\text { NeuroInsight } \\
\text { www.neuro-insight.com }\end{array}$ & $\begin{array}{l}\text { Melbourne, } \\
\text { AUSTRALIA }\end{array}$ & $\begin{array}{l}\text { Dr. Richard } \\
\text { Silberstein }\end{array}$ & EEG & $\begin{array}{l}\text { Utiliza una modificación } \\
\text { patentada de la EEG: Steady- } \\
\text { State Topography }\end{array}$ \\
\hline $\begin{array}{l}\text { LABoratory. } \\
\text { www.labtd.com }\end{array}$ & $\begin{array}{l}\text { Varsovia, } \\
\text { POLONIA }\end{array}$ & $\begin{array}{l}\text { Dr. Rafal } \\
\text { Ohme. }\end{array}$ & $\begin{array}{l}\text { EEG, Electro- } \\
\text { miografía, EDA }\end{array}$ & $\begin{array}{l}\text { Colabora con MSW Research } \\
\text { en EE.UU. Su sistema se } \\
\text { denomina Neuro-Trace. }\end{array}$ \\
\hline $\begin{array}{l}\text { MindLab International, } \\
\text { www.themindlab.org }\end{array}$ & $\begin{array}{l}\text { Sussex, } \\
\text { REINO } \\
\text { UNIDO }\end{array}$ & $\begin{array}{l}\text { Dr. David } \\
\text { Lewis- } \\
\text { Hodgson }\end{array}$ & $\begin{array}{l}\text { EEGq }^{13}, \text { eye- } \\
\text { tracking, EMG, } \\
\text { EDA, otras. }\end{array}$ & $\begin{array}{l}\text { Sistema propietario de medi- } \\
\text { ción denominado Neurome- } \\
\text { trix }{ }^{2} \text {. }\end{array}$ \\
\hline $\begin{array}{l}\text { NeuroSense, } \\
\text { www.neurosense.com }\end{array}$ & $\begin{array}{l}\text { Londres, } \\
\text { REINO } \\
\text { UNIDO }\end{array}$ & $\begin{array}{l}\text { Dr. Gemma } \\
\text { Calvert }\end{array}$ & $\begin{array}{l}\text { fMRI, EEG, } \\
\text { EDA. }\end{array}$ & $\begin{array}{l}\text { No forma parte de la iniciativa } \\
\text { de estandarización de la ARF. }\end{array}$ \\
\hline $\begin{array}{l}\text { TNS Magasin, } \\
\text { www.tnsmagasin.com }\end{array}$ & $\begin{array}{l}\text { Londres, } \\
\text { REINO } \\
\text { UNIDO }\end{array}$ & $\begin{array}{l}\text { Cristina de } \\
\text { Balanzó }\end{array}$ & $\begin{array}{l}\text { EEG, eye- } \\
\text { tracking }\end{array}$ & $\begin{array}{l}\text { Empresa de investigación y } \\
\text { consultoría enfocada a tiendas. } \\
\text { Tienen un departamento de } \\
\text { neurociencia. No es una con- } \\
\text { sultora de neuromarketing } \\
\text { propiamente dicha. }\end{array}$ \\
\hline
\end{tabular}

La tabla cubre a los principales proveedores internacionales en el mercado del neuromarketing. En España tenemos dos casos interesantes. Por un lado, Neurobiomarketing $^{14}$, con oficinas en Milán y Barcelona, también ofrece servicios de neuromarketing a nivel internacional. Su sistema de medición (Mente Corazón Ojos Manos) utiliza tecnologías como el EEG, eye-tracking o el registro de la EDA para analizar las reacciones de los sujetos a los spots publicitarios (y otras piezas). En colaboración con McCann Erickson España y Universal McCann ha creado una

\footnotetext{
$12 \mathrm{http}: / /$ www.gallup-robinson.com/services/cera.pdf

13 Mindlab utiliza una versión específica del EEG llamada encefalografía cuantificada.

$14 \mathrm{http} / / / \mathrm{www}$.neurobiomarketing.com/
} 
base de datos con las reacciones de más de 400 sujetos a distintas piezas audiovisuales. Neurobiomarketing dispone de varios algoritmos propios (pendientes de patente) para el análisis de piezas publicitarias y están continuamente experimentando con diferentes tecnologías. Los dos socios fundadores de Neurobiomarketing son Brian Loeb y Alberto Apicella.

Por otro lado, ICON Multimedia ${ }^{15}$ está desarrollando una versión avanzada del Sociograph, un instrumento de medición psicobiológica que registra la EDA de un grupo de sujetos sometidos a un estímulo (Martínez Herrador, Garrido Martín et al. 2010). Diseñado inicialmente por el catedrático de la Universidad de Salamanca José Luis Martínez Herrador para analizar las reacciones de los jurados durante las declaraciones en los juicios, el Sociograph aprovecha un efecto colateral de la medición simultánea de un grupo. Las pequeñas activaciones electrodérmicas dependientes de características personales de los sujetos se ven compensadas por el resto del grupo, a la vez que las reacciones provocadas por estímulos externos se «suman» entre ellas y se vuelven más evidentes. ICON Multimedia ha desarrollado un prototipo mejorado de la tecnología diseñada por Martínez Herrador para que esta sea wireless y permita libertad de movimientos a los hasta 128 sujetos estudiados simultáneamente.

Las distintas empresas de este mercado ofrecen una variedad de servicios. Un servicio que destaca entre los demás es el copytesting de spots publicitarios. Las grandes marcas invierten mucho dinero en la producción y difusión de piezas audiovisuales cortas. Una mejora en su efectividad puede traerles un incremento en el retorno de inversión; pero para que el neuromarketing sea una opción viable estos beneficios tienen que ser suficientes para justificar los de 10.000 a 20.000 euros que pueden costar los estudios más sencillos. Por lo tanto, el uso de técnicas de neuromarketing en pretest de anuncios es algo que normalmente solo está al alcance de grandes marcas con presupuestos publicitarios elevados.

Las piezas audiovisuales se prestan bien al análisis mediante técnicas de neuromarketing porque se las puede aislar como estímulo único, medir las reacciones de los sujetos expuestos y luego agrupar los datos. El estímulo audiovisual se puede utilizar como «sincronizador cognitivo» y se ha demostrado que las respuestas son bastante estereotípicas entre diferentes sujetos cuando la actividad monitorizada es la observación de la misma pieza (Hasson, Nir, et al., 2004). El neuromarketing podría utilizarse durante el proceso montaje del spot publicitario para elegir entre los diferentes planos aquéllos que mayor respuesta emocional o mayores posibilidades de recuerdo (Hasso, Furman, 2008) generen. Los creativos y realizadores ofrecerían distintas posibilidades a la consultora y ella se encargaría de determinar cuál de las versiones tiene mayor impacto en el consumidor de acuerdo a sus respectivas metodologías. 
Además de la pieza en sí, a veces también se estudia el efecto que produce el contexto (normalmente el programa en el que se inserta) en la recepción de un anuncio. Los descubrimientos iniciales parecen indicar que un GRP (gross rating point) no siempre es un GRP. Es decir, que la reacción cerebral al anuncio podría variar dependiendo del programa durante el cual éste se emita ${ }^{16}$. La consolidación de un descubrimiento como este podría tener grandes consecuencias sobre la disciplina publicitaria de planificación de medios.

Aparte de los publicistas, los productores de cine también podrían tener muy buenos motivos para utilizar estas técnicas de investigación. En las producciones norteamericanas, la película en sí puede costar unos 100 millones de dólares, más otro tanto gastado en marketing. Suena razonable invertir en mejorar el producto antes de exponerlo en las salas. Del mismo modo que en el copytesting de spots publicitarios, podría utilizarse el neuromarketing para seleccionar entre distintos planos o secuencias en el montaje final de la película.

Quitando el análisis de piezas audiovisuales (televisión o cine), a partir de las páginas web de las empresas que figuran en la tabla anterior, podemos resumir los servicios más habituales ofrecidos por las consultoras:

- Consultoría de Marca. Con distintos servicios basados en la medición de la evaluación inconsciente de la marca por parte de los sujetos de estudio, comparaciones entre marcas o sistemas de selección de los personajes adecuados a contratar para representar a la marca.

- Diseño de Producto. Distintos servicios basados en analizar la experiencia del consumidor en la interacción con el producto, su respuesta al packaging, la evaluación de olores...

- Investigación en Tienda. Orientada a comprender los procesos de compra de los consumidores y mejorar la organización de las superficies comerciales.

- Análisis de Websites. Detección de áreas de interés, emociones generadas, tareas difíciles/fáciles, barreras para la compra, usabilidad desde el punto de vista neurológico...

- Test de Videojuegos. Evaluación de la calidad de la experiencia, comparación con la competencia, detección de elementos especialmente atractivos...

- Fijación del Precio Óptimo. Se analiza cuál es el precio óptimo desde un punto de vista neurológico, saltándose los errores que pudieran introducir los intereses de los entrevistados mediante medios convencionales.

- Servicios orientados al Marketing Político. Aunque no son muy diferentes a los ofrecidos para otros sectores (copytesting de anuncios electorales, análisis de reacción a discursos...), a veces se los considera una categoría aparte por sus implicaciones para la opinión pública.

16 Según lo presentado por Bartlomiej Serafinski y Janusz Plencler en Neuroconnections 2009 (Cracovia, 5-7 Feb.). Sin documentación disponible en Internet o publicada en revistas científicas. 
La investigación en tienda (grandes superficies normalmente), la evaluación de páginas web o incluso los estudios para ayudar en el proceso de diseño de productos tienen un problema que el copytesting de piezas audiovisuales no tiene: el recorrido que realiza un comprador en una tienda o página web no está predeterminado y por lo tanto es diferente para cada sujeto. Del mismo modo, no se puede prever cómo será la interacción entre el producto y el consumidor: cada uno examinará el objeto de manera diferente y en un orden diferente. Resulta complicado y trabajoso agregar esos datos para poder separar lo personal de las reacciones que se repiten y suponen un indicio de tendencia.

Se han utilizado distintos acercamientos para solucionar este problema. Una oferta habitual de algunas empresas de investigación es testar por separado imágenes o videos que formen parte de la pieza. Así por ejemplo, se puede presentar un «pantallazo» de la home de una página web para ver la reacción emocional del usuario o analizar individualmente los videos que se insertarán en la web. También se pueden estudiar por separado displays de tienda o fotos de los lineales de supermercado, aunque el entorno se aleje mucho de la situación real en tienda.

Otra forma de afrontar al problema, más costosa pero quizá más interesante, es combinar EEG (u otras tecnologías) con eye-tracking y realizar un análisis manual posterior con todos los datos recogidos (De Balanzó, Serrano Abad, et al., 2010). De este modo, el investigador obtiene la información sobre lo que estaba mirando el sujeto y la reacción que se generaba en su cerebro en cada momento. Después, todos esos datos son procesados por investigadores entrenados para ser convertidos en conclusiones útiles.

\section{Principales debates y retos en el mercado del neuromarketing}

\section{1. ¿Funciona?}

Como hemos dicho, se ha demostrado científicamente que la terminología procedente de las neurociencias tiene una capacidad persuasiva superior (Weisberg, Keil, et al., 2008). Esto ha llevado a numerosos periodistas y profesionales del marketing a hablar sobre el neuromarketing de manera simplificada, exagerada o simplemente errónea. Como consecuencia, existe un cierto escepticismo entre muchos científicos sobre la viabilidad de estas técnicas.

La aplicabilidad de las tecnologías de las neurociencia a la investigación de mercado ha sido analizada en varios artículos (Ariel, Berns, 2010; Kenning, Plassmann, 2008; Lee, Broderick et al., 2007) a partir de la literatura científica disponible. Las conclusiones de estas revisiones apuntan a que las neurociencias pueden utilizarse para resolver algunas cuestiones tanto de la economía como de la investigación de mercados.

Se han publicado estudios en revistas científicas revisadas por pares en los que la neurociencia se utilizaba para identificar el efecto de la marca sobre la experien- 
cia de consumo del producto (McLure. Li et al., 2004), para averiguar qué mecanismos neurales están relacionados con la publicidad emocional (Ambler, Ioannides et al., 2000), para arrojar luz sobre el proceso de elección de marca/producto (Deppe, Schwindt et al., 2005; Plassmann, Kenning et al., 2007; Knutson, Rick et al, 2007) o para determinar si, cuando evaluamos la personalidad de una marca, intervienen factores similares a los que entran en juego cuando lo hacemos con la personalidad de una persona (Yoon, C.; Gutchess, 2006).

La cuestión fundamental continúa siendo si el neuromarketing puede ayudar a crear anuncios más efectivos $\mathrm{y}$, aunque en esa línea podemos consultar varios estudios realizados por científicos de LAB sobre el uso de EEG, EDA y electromiografía para evaluar la respuesta a la publicidad (Ohme, Reykowska et al., 2009; Ohme, Reykowska et al., 2010), dichas investigaciones no han dejado de recibir críticas en el aspecto metodológico ${ }^{17}$. Nos faltan evidencias para saber si la aplicación de estas nuevas técnicas efectivamente mejorará la práctica publicitaria.

Ni las metodologías que utilizan las principales consultoras de neuromarketing ni sus resultados han trascendido aún a las revistas científicas, ya que obviamente son una ventaja competitiva en este mercado. Por lo tanto, nos queda esperar para responder definitivamente a esta cuestión desde un punto de vista científico, pero desde luego hay indicios para ser optimistas si tenemos en cuenta las conclusiones de los estudios que se han citado.

\subsection{Costes}

El uso de tecnología de las neurociencias para la investigación de marketing es a día de hoy una opción cara. El uso de tecnología fMRI puede suponer un costes superiores a 550 dólares/hora, lo que hace que los científicos se pregunten si el empleo de estas técnicas, que aún no han demostrado ser capaces de obtener mejores resultados que las metodologías convencionales, es justificable desde el punto de vista económico ${ }^{18}$.).

El sector del neuromarketing ha tomado diferentes vías para mantener los costes a niveles controlables. Por un lado, muchas consultoras utilizan tecnologías como la EEG, que es bastante más barata que la fMRI o la MEG. Las limitaciones de la EEG para acceder a la información de fenómenos que suceden en capas más profundas del cerebro ya se han tratado anteriormente.

Por otro lado, la mayor parte de los estudios de neuromarketing utilizan muestras reducidas alegando mayor sensibilidad de estas técnicas y conclusiones basadas en automatismos cerebrales más o menos universales. Este tema se tratará más en profundidad en el siguiente punto, pero, como es evidente, la muestra tiene gran

17 ARIEL, BERNS (2010), op. cit. 288.

18 Ibidem, 287; RoBINSON, J. (2006): «Is That a Neuromarketer in Your Brain?» Gallup Management Journal, January 12. http://gmj.gallup.com/content/20785/neuromarketer-your-brain.aspx. Web visitada el $19 / 02 / 2011$. 
influencia tanto sobre el coste de la investigación como sobre los tiempos en los que esta puede realizarse. El acceso a un solo equipo de medición, por ejemplo, ralentiza mucho la adquisición de datos, mientras que disponer de varios equipos y profesionales que puedan manejarlos simultáneamente dispara los costes.

No hay estudios que analicen el retorno de inversión del neuromarketing y, por lo tanto, es difícil desde el punto de vista de los responsables de marketing decidirse sobre la conveniencia de utilizarlo. Sí parece evidente que las empresas que más tienen que ganar por el empleo de estas técnicas son las mismas que pueden invertir $10.000 €$ 20.000 euros para pretestar un anuncio de televisión antes de emitirlo. Son grandes empresas que ya emplean habitualmente encuestas, focus groups, entrevistas en profundidad y otras técnicas de investigación para orientar sus decisiones. Para estas empresas, una nueva forma de abordar el problema puede ofrecer insights que hasta el momento permanecían ocultos en el subconsciente de sus públicos.

\subsection{Tamaño de la muestra}

Una de las grandes discusiones del sector tiene que ver con el tamaño de la muestra de los estudios de neuromarketing. El alto coste de la tecnología y la supuesta similitud entre las respuestas neurológicas de los sujetos hacen que las empresas de este sector estén trabajando con muestras realmente pequeñas. Mientras que los estudios de mercado mediante encuestas requieren cientos de respuestas, consultoras de neuromarketing como Sands Research aseguran que es suficiente con una muestra de 30-40 sujetos por cada grupo demográfico para obtener resultados con un margen de $1 \%$ de error con la tecnología EEG (Sands, 2009). La mayor parte de las consultoras que emplean EEG trabajan con muestras de ese tamaño o, dependiendo del margen de error que quieran permitirse, incluso con muestras más pequeñas.

No faltan voces críticas con esta situación. EmSense, consultora que reivindica que hace «neuromarketing cuantitativo», considera que se necesitan muestras de mayor tamaño, equivalentes a las que se utilizan en otras áreas de la investigación de mercados. Para conseguirlo, ha diseñado sus EmBand con una cantidad menor de electrodos (dos frente a los 32-64 que se utilizan habitualmente) que registran la actividad en zonas muy concretas. Recogiendo menos información (o información más concreta), pueden producir equipos más baratos e incrementar el tamaño de la muestra sin disparar los costes.

Eso sí, los electrodos que EmSense utiliza en sus EmBands no requieren gel para utilizarlos (son electrodos «secos»), por lo que otros profesionales del neuromarketing les han respondido a su vez con críticas ${ }^{19}$, ya que la tecnología EEG más moderna utiliza electrodos humedecidos con un gel especial para evitar que las activaciones musculares produzcan interferencias. Scott Smith, de EmSense, ha defendido la posición de su compañía haciendo referencia a varios artículos científicos (Estepp, Christensen et al. 2009; 
Taheri, Knight et al., 1994) que sugieren que los electrodos secos podrían ser tan efectivos como los húmedos, aunque uno de ellos está basado en una muestra de tan sólo 6 sujetos y el otro, aunque coescrito por un respetable neurocientífico, Robert T. Knight, tiene más de 15 años. El Dr. Knight trabaja en la actualidad para consultora Neurofocus, que investigaba bajo los mismos estándares (muestras reducidas, electrodos húmedos que cubren todo el cuero cabelludo) que Sands Research, LAB, The Mindlab o Neuro-Insight hasta hace muy poco. El 21 de marzo de 2011, Neurofocus reveló una nueva tecnología diseñada para llevar la recogida de datos EEG a los hogares de centenares de sujetos (construyendo paneles que optimicen costes). Este aparato, denominado Mynd, utiliza electrodos secos para ser compatible con el uso doméstico ${ }^{20}$.

En cualquier caso, ninguno de los actores actuales del mercado del neuromarketing niega la idoneidad de incrementar el tamaño de la muestra, especialmente cuando hay varios grupos demográficos implicados o se quieren hacer comparaciones entre subgrupos. No olvidemos que en el ámbito académico se están oyendo ya voces de alarma (Henrich, Heine et al., 2010) a consecuencia de la generalización a la población general de resultados de investigaciones que normalmente tienen una muestra de un subgrupo poblacional muy concreto, denominado WEIRD (acrónimo en inglés de occidental, educado, industrializado, rico y democrático). Los estudios de neuromarketing, con sus muestras reducidas, podrían estar multiplicando aún más las consecuencias de este sesgo.

No obstante, en el mercado la sostenibilidad manda. A día de hoy y para grupos demográficos concretos, una muestra de 30-50 personas es la mejor opción coste/resultados, por lo menos si nos referimos a tecnología de EEG.

\subsection{Necesidad de estándares}

Uno de los principales problemas del sector del neuromarketing es la ausencia de estándares ampliamente aceptados por los distintos agentes. Cada consultora tiene su propia metodología: registra datos de manera diferente, con distintas tecnologías y los analiza en base a criterios diferentes. De este modo, dos empresas distintas pueden estar refiriéndose a la emoción que genera un determinado plano en los espectadores y ofrecer un valor numérico de esa emoción (un índice con un número positivo o negativo). Pero ambos valores numéricos no serán comparables puesto que habrán sido obtenidos mediante procesos completamente distintos (y probablemente tengan incluso magnitudes distintas). No se ha hecho ningún intento público de comparar sistemas como Neuro Engagement Score (Sands Research), Neurotrace (Lab), Neurometrix (Mindlab) y otros sistemas propietarios.

No hay manera de que un cliente pueda contratar un estudio de una misma pieza a dos compañías y comparar los resultados, ya que manejan índices y tecnologías

$20 \mathrm{http}: / /$ neurogadget.com/2011/03/21/neurofocus-reveals-mynd\%E2\%84\%A2-the-first-wireless-fullbrain-eeg-headset/1416 
diferentes. Es todavía más complicado comparar los resultados de piezas publicitarias diferentes. Si esto fuera posible, permitiría a una empresa, por ejemplo, ir probando año a año el trabajo de distintas consultoras. Esta circunstancia supone un freno importante para el desarrollo del sector.

Se están haciendo algunos intentos por establecer estándares. La Advertising Research Foundation (ARF) ${ }^{21}$ norteamericana ha puesto en marcha una iniciativa denominada Neurostandards Collaboration ${ }^{22}$ y ha reunido a ocho de los principales actores del mercado: Gallup \& Robinson, Innerscope, LABoratory, Mindlab International, NeuroCompass, Neuro-Insight, Sands Research y Sensory Logic. Las conclusiones de esta primera fase se publicaron el 22 de marzo de $2011^{23}$ y han sido muy limitadas. Aunque reconocen que las distintas técnicas analizadas (incluyendo fMRI, EEG, FACS, EDA, electromiografía...) pueden ser útiles en la investigación de mercados, también señalan que la publicidad está basada en estímulos complejos (imágenes, palabras y música que cambia rápidamente) y que resulta difícil separar qué genera cada reacción. Específicamente apuntan varios problemas:

- Las imágenes tienen tiempos de reacción distintos (el cerebro reacciona más rápido ante un peligro que ante una imagen placentera).

- Las reacciones a determinados estímulos pueden estar condicionadas por estímulos anteriores.

- Medir la atención resulta menos complejo que determinar emociones específicas o la intención de compra del sujeto.

Se espera que se publiquen los resultados de una segunda fase denominada Neurostandards 2.0 en un detallado white paper para final del verano de 2011.

Pero las tensiones de mercado siguen muy presentes. Dos de las principales empresas norteamericanas de neuromarketing, Neurofocus y EmSense, no participan de esta iniciativa. Neurofocus, de hecho, anunció la publicación de sus propios estándares (NeuroStandards) poco después de que la ARF anunciara su iniciativa (Neurofocus, 2010), con lo que es muy posible que aún tengamos bastante que esperar antes de ver un estándar claro en el mercado, sea éste acordado entre empresas o fijado por imposición del jugador más fuerte.

\section{5. Ética}

Uno de los debates más encendidos en este mercado es el que tiene que ver con la ética de la investigación con estas tecnologías. En general y como mecanismo defensivo, la práctica totalidad de las consultoras de neuromarketing están de acuerdo en que deben adherirse a los más altos principios éticos. En un mercado tan dispar, con tan

\footnotetext{
$21 \mathrm{http}: / /$ www.thearf.org/

$22 \mathrm{http}: / / \mathrm{www}$.thearf.org/assets/neurostandards-collaboration

23 http://www.thearf.org/assets/pr-2011-03-21
} 
pocos estándares y con tanta competencia, resulta curioso que las empresas se pongan de acuerdo en algo. La razón por la que esto es así es la alarma social que tiende a generar el neuromarketing cuando la información relativa a él llega a los medios. Las generalizaciones de los periodistas (y a veces de los propios consultores de neuromarketing) y el miedo a «ser controlados» por esta nueva tecnología tienen como consecuencia que las declaraciones de comportamiento ético por parte de las consultoras sean una medida habitual para calmar a la opinión pública.

Es necesario señalar que las investigaciones de neuromarketing se acogen habitualmente a los mínimos éticos de la investigación de mercados. Por ejemplo, a las recomendaciones contenidas en el Código de Estándares en Investigación de Mercados (Marketing Research Asociation, 2007). No obstante, la discusión se centra en si son necesarias normas éticas adicionales relacionadas con la especificidad de la tecnología.

Varios autores han intentado resumir algunos de los problemas éticos específicos que podría traer el uso del neuromarketing ${ }^{24}$ :

- Privacidad en las preferencias y «lectura mental». Los sujetos de investigación deberían ser capaces de elegir qué información revelan acerca de sus preferencias o sus pensamientos. En cierto modo, el neuromarketing les quita esa elección al tomar las respuestas directamente de su actividad cerebral, aunque la profundidad real de la información que actualmente puede accederse no debería generar grandes preocupaciones. Existe un intenso debate acerca de la capacidad actual de la tecnología para «leer los pensamientos» de la gente y cómo debería reaccionar la sociedad si esa capacidad se incrementase en un futuro cercano (Kulynych, 2002; Illes and Racine, 2005; Tovino, 2005; Greely, 2006; Alpert, 2007; Appelbaum, 2007; Illes, 2007; Tovino, 2007).

- La información puede usarse para afectar a personas individuales o subgrupos que compartan un rasgo neurológico. La información podría utilizarse para explotar «debilidades» individuales o de pequeños grupos. Es necesario hacer una reflexión ética adicional sobre las medidas protectoras a tomar en los casos en los que una «vulnerabilidad» neurológica sea detectada y pueda ser explotada comercialmente con subgrupos determinados.

- Ruta central o periférica. La información obtenida en el neuromarketing se puede utilizar para mejorar mensajes dirigidos a la ruta periférica de procesamiento (Petty, Cacciopo, 1986), más relacionada con factores inconscientes, lo que algunos consideran éticamente cuestionable. Sin embargo, con respecto a esto hemos de tener en cuenta que los profesionales de la publicidad llevan dirigiendo sus mensajes a esa ruta periférica desde hace décadas sin ayuda del neu-

24 Ariel, Berns (2010), op. cit. 289; MuRPhy, E.R,; Illes, J.; ReINER, P.B. (2008): «Neuroethics of neuromarketing», Journal of Consumer Behaviour, 7, 293-302. 
romarketing, por lo que el revuelo en torno a estas tecnologías no estaría justificado en proporción.

- Descubrimientos anormales. Las anomalías en la fMRI ocurren aproximadamente en el $1 \%$ de los casos. Muchas veces puede ser un simple falso positivo, pero en otras ocasiones puede suponer la detección de un problema de salud para el sujeto estudiado. Las empresas de neuromarketing deberían tener una política escrita para actuar en esos casos, basándose quizá en lo que investigadores clínicos han acordado previamente (Illes, Kirschen et al., 2006).

- Ausencia de regulación específica. Las investigaciones de neuromarketing deberían ser revisadas por algún tipo de entidad independiente que en estos momentos no existe. En EE.UU., por ejemplo, al no ser investigación clínica, no existe una Institutional Review Board que imponga unas normas mínimas.

- Representación mediática ajustada a la realidad. No es infrecuente comprobar que las consultoras exageran las capacidades del neuromarketing. En general, toda esa exageración ha ido en su detrimento, provocando reacciones negativas en la opinión pública. Desde un punto de vista ético, debe considerarse que es posible que la exageración tenga como consecuencia pérdidas económicas para las empresas que decidan utilizar estas técnicas de investigación atraídas por promesas que no pueden cumplirse y, por lo tanto, es fundamental que el sector invierta esfuerzo en que los medios ofrezcan una visión ajustada a la realidad del neuromarketing. La prestigiosa revista Nature Neuroscience ha dedicado varias editoriales al tema (Editorial, 2004; Editorial, 2007), dada la importancia que esto puede tener para el propio prestigio de la ciencia.

- Podría utilizarse la tecnología para desarrollar técnicas de manipulación subliminal. Existen ciertas evidencias de que podemos percibir estímulos e incluso que esos estímulos pueden tener efectos sobre nuestras conductas (Aarts, Custers et al., 2008). Con la tecnología actual no es factible que esos indicios puedan convertirse en estrategias infalibles de venta, pero queda la duda de qué sucedería si tales técnicas de venta llegaran a desarrollarse (Murphy, Illes et al., 2008).

- Las empresas no tendrán en mente el interés del consumidor. A pesar de que el marketing pretenda combinar apropiadamente los objetivos de las empresas y los consumidores, sabemos que éstas a veces se guían por los resultados a corto plazo, por lo que nos corresponde diseñar códigos éticos pensando en esta circunstancia. No obstante, esta reflexión no es válida únicamente para el neuromarketing.

En cualquier caso, gran parte de la alarma social proviene más del desconocimiento sobre las aplicaciones reales del neuromarketing que de los problemas reales que puedan acarrear estas tecnologías. Del mismo modo que la publicidad 
subliminal y el libro The Hidden Persuaders de Vance Packard ${ }^{25}$ crearon revuelo social injustificado en 1957, los consumidores de hoy día han considerado que el neuromarketing tiene mucha mayor capacidad de manipulación en comparación con otras estrategias comerciales que no hacen uso de las neurociencias, lo que en el fondo es falso en la mayoría de los casos (Levy, 2009).

Por el momento, la información que provee el neuromarketing es muy similar a la que proveen otras técnicas de investigación de mercados (preferencias, elementos clave) con una notoria excepción. En muchos casos, el sujeto no sabe qué información está revelando porque él mismo no es consciente de sus preferencias. Ofreciendo adecuada información a los sujetos de investigación sobre los datos que se van a recoger y el propósito que se les va a dar, así como teniendo cuidado en la manipulación de datos personales o de información desagregada (las reacciones de una persona individual al estímulo) y desarrollando un protocolo de actuación para los hallazgos anormales, la investigación con técnicas de neuromarketing no debería ofrecer mayores problemas éticos que la investigación de mercados convencional.

\section{Conclusiones}

El mercado del neuromarketing se presenta en su infancia, con algo más de una docena de actores internacionales (la mayoría en EE.UU.) que pugnan entre sí por establecer un estándar de investigación de mercados basado en neurociencias. Las dos tecnologías realmente «neuro» más utilizadas son la fMRI y la EEG, con una clara preferencia por esta segunda en el ámbito comercial a consecuencia de los costes. No obstante, la mayor parte de consultoras utilizan una combinación de tecnologías de medición biométrica (respuesta galvánica de la piel, electromiografía, eye-tracking, ritmo cardiaco...) para complementar los resultados obtenidos por la EEG y, a veces, ponerlos en contexto (como en el caso de la investigación en tienda con eye-tracking).

Independientemente de la tecnología utilizada para recoger los datos, cada consultora tiene su propia metodología de análisis. Existen tímidos intentos de establecer un estándar para el mercado, pero aún distan mucho de haber fructificado, por lo que podemos encontrarnos en la actualidad con debates como cuál es el tamaño de muestra apropiado para estas técnicas o si es mejor utilizar electrodos húmedos o secos.

Los servicios concretos que se ofrecen varían mucho entre una consultora y otra, pero el test previo a la emisión de piezas audiovisuales es el servicio estrella de casi todas ellas, por lo que la industria del cine y la publicidad son los clientes más lógicos. El neuromarketing tiene también aplicaciones en política, diseño de videojuegos, organización de puntos de venta, packaging o diseño web, entre otras. 
Cada consultora guarda celosamente sus métodos de investigación. El sector no dispone de metodologías ampliamente aceptadas por la comunidad científica y el número de experimentos publicados en revista académicas aún es limitado como para resolver la cuestión fundamental: ¿puede el neuromarketing ayudar a mejorar la comunicación de las empresas? En cualquier caso, los estudios publicados nos permiten ser optimistas acerca de las posibilidades de la tecnología, aunque los costes de utilizarla haga que de momento solo sea una opción atractiva para las empresas con mayor presupuesto publicitario.

Uno de los debates principales del sector es el que tiene que ver con la ética. Por una parte, el uso de las tecnologías trae consigo una serie de posibles problemas éticos, como es el descubrimiento accidental de anomalías que puedan indicar alguna patología o el descubrimiento de preferencias de las que el sujeto ni siquiera es consciente y no querría revelar. La mayor parte de las consultoras están de acuerdo en este punto: que es necesario adherirse a los estándares éticos más altos ofreciendo toda la información que sea necesaria a los sujetos de estudio.

Por otra parte, las consultoras de neuromarketing deberían adquirir el compromiso ético de suministrar a los medios de comunicación información veraz y ajustada a la realidad. La exageración sobre las posibilidades del neuromarketing por parte de los periodistas es la principal culpable de la alarma social que se ha generado en torno estas técnicas, a las que se asocia con el control mental, la manipulación y la publicidad subliminal. En este punto, el comportamiento de las consultoras de neuromarketing varía, ya que la competencia por hacerse con esos primeros clientes innovadores de este mercado es feroz. Más de una de ellas se ha dejado llevar por la necesidad de atraer nuevos clientes y ha realizado afirmaciones con escasa base científica a los medios de comunicación, lo que va en detrimento tanto del mercado como del prestigio de la propia ciencia en general.

En conclusión, en los próximos años el mercado del neuromarketing estará en posición de ofrecer avances científicos y profesionales muy interesantes, aunque de momento ofrece más promesas que aplicaciones reales válidas para la mayor parte de las empresas. Mantener un ojo en él sin dejar de hacer lo que estés haciendo es la mejor recomendación que se puede dar a los profesionales de la comunicación. La mejor manera de seguir los hitos de este mercado probablemente la ofrecen blogs especializados en el tema, como Neuromarketing ${ }^{26}$ (de Rooger Dooley), Neurobusiness ${ }^{27}$ (Mitra-Khan y Levallois), Brainethics ${ }^{28}$ (Thomas Ramsoy) o Neuromarca $^{29}$ (escrito en español por uno de los autores de este artículo). Para los científicos, sin embargo, el neuromarketing es un campo de conocimiento lleno de 
oportunidades de investigación y de descubrimientos por hacer. Lo que suceda en estos años sentará las bases del conocimiento en este campo.

\section{Referencias bibliográficas}

AARTS, H.; Custers, R.; MARIEN, H. (2008): «Preparing and Motiating Behaviour Outside Awareness», Science, 319, 1639.

ALPERT, S. (2007): «Brain privacy: how can we protect it?», The American Journal of Bioethics, 7, 70-73.

AMBLER, T.; IOANNIDES, A.; RoSE, S. (2000): «Brands on the brain: Neuro- images of advertising», Business Strategy Review, 11(3), 17-30.

APPELBAUM, PS. (2007) «Law \& psychiatry: the new lie detectors: neuroscience, deception, and the courts», Psychiatric Services, 58, 460-462.

ARIEL, D.; BERNS, G.S. (2010): «Neuromarketing: the hope and hype of neuroimaging in business», Nature Reviews Neuroscience, April 2010, 11.

Azcarate, A.; Hageloh, F.; SANDE, K.; Valenti, R. (2005): «Automatic facial emotion recognition». Tech report. University of Amsterdam, http://staff.science.uva.nl/ rvalenti/projects/mmis/Automatic\%20Facial\%20Emotion\%20Re cognition.pdf. Web consultada el 18/07/2011.

BERNS, G.S.; MOORE, S.E. (2010): «A neural predictor of Cultural Popularity». Available at SSRN: http://ssrn.com/abstract=1742971

BoucSEIN, W. (1992): Electrodermal activity, New York, Plenum Press.

BRAIDOT, N. (2009): Neuromarketing, Barcelona, Gestión 2000.

CRANDALL, K.B. (2006): «Invisible Commercials and Hidden Persuaders: James M. Vicary and the Subliminal Advertising Controversy of 1957», Undergraduate Honors Thesis. University of Florida. Available at http://plaza.ufl.edu/cyllek/docs/KCrandall_Thesis2006.pdf

Coutler, R.A.; Zaltman, G.; CoutLeR, K.S. (2001): «Interpreting Consumer Perceptions of Advertising: An Application of the Zaltman Metaphor Elicitation Technique», Journal of Advertising, 30(4), Winter 2001.

Davidson, R. J., Ekman, P., SAron, C. D., Senulis, J. A.; Friesen, W. V. (1990): «Approach-withdrawal and cerebral asymmetry: emotional expression and brain physiology», Journal of Personality and Social Psychology, 58, 330-341.

De BAlanzó, C.; SerRano Abad, N.; SCAMEll-KatZ, S. (2010): «Damasio: a starting point for integrating neuroscience findings into retail research», comunicación presentada en ESOMAR Congress 2010, https://www.esomar.org/web/publication/paper.php?id=2153, Web visitada el 21/02/2011.

DepPe, M.; Schwindt, W.; Kugel, H.; Plassmann, H.; Kenning, P. (2005): «Non-Linear responses within the medial prefrontal cortex reveal when specific implicit information influences economic decision-making», Journal of Neuroimaging, 15(2), 171-183.

Deppe, M.; Schwindt, W.; PiePer, A.; Kugel, H.; Plassmann, H.; Kenning, P.; Deppe, K.; RINGELSTEIN, E.B. (2007): «Anterior cingulate reflects susceptibility to framing during attractiveness evaluation», Neuroreport, 18(2), 1119-1123.

EDITORIAL (2004): «Brain scam?», Nature Neuroscience, 7, 683.

- (2007): «Mind games», Nature, 450, 457.

EkMAN, P.; FrIeSEN, W. (1978): Facial Action Coding System: A Technique for the Measurement of Facial Movement, Palo Alto, Consulting Psychologists Press. 
EstePP, J. R.; Christensen, J.C.; Monnin, J.W.; DAVIS, I.M.; Wilson, G.F. (2009): «Validation of a Dry Electrode System for EEG», Human Factors and Ergonomics Society Annual Meeting Proceedings, 53(18), 2009, 1171-1175.

GREELY, HT. (2006): «The social effects of advances in neuroscience: legal problems, legal perspectives», en J Illes(ed), Neuroethics: Defining the Issues in Theory, Practice, and Policy, Oxford University Press, Oxford, 245-264.

GROEPPEL-KLEIN, A. (2005): "Arousal and consumer in store behavior», Brain Research Bulletin, 67, 428-437.

GROSENICK, L.; GREER, S.; KNUTSON, B. (2008): «Interpretable classifiers for FMRI improve prediction of purchases», IEEE Transactions on Neural Systems and Rehabilitation Engineering. 16, 539-548.

Hare, T. A., O’Doherty, J., CAMERER, C. F., Schultz, W.; RANGel, A.(2008): «Dissociating the role of the orbitofrontal cortex and the striatum in the computation of goal values and prediction errors», Journal of Neuroscience, 28, 5623-5630.

HARMON-JONES, E. (2003): «Clarifying the emotive functions of asymmetrical frontal cortical activity», Psychophysiology 40, 838-848.

HASSON, U.; NIR, Y.; LEVY, I.; FUHRMANN, G.; MALACH, R. (2004): «Intersubject synchronization of cortical activity during natural vision», Science, 303, 1634-1640.

HASSON, U.; FURMAN, O.; ClARK, D.; DUDAI, Y.; DAVACHI, L. (2008): «Enhanced intersubject correlations during movie viewing correlate with successful episodic encoding», Neuron, 57, 452-462

Henrich, J.; HeIne, S.J.; CNOREZAYAn, A. (2010): «The weirdest people in the world?», Behavioral and Brain Sciences, 33, 61-135.

Huster, R. J., Stevens, S., Gerlach, A. L. \& Rist, F. (2008): «A spectroanalytic approach to emotional responses evoked through picture presentation», International Journal of Psychophysiology, 72, 212-216.

Illes, J.; KIrSchen, M.P.; EdWARds, E.; StANFORD, L R.; BANDETTINI, P; CHO, M.K.; ForD, P.J.; Glover, G.H.; Kulynych, J.; Macklin, R.; Michael, D.B; Wolf, S.M. et al. (2006): «Incidental Findings in Brain Imaging Research», Science, 311, 783-784.

ILLES, J. (2007): «Empirical neuroethics. Can brain imaging visualize human thought? Why is neuroethics interested in such a possibility?», EMBO Reports 8 (Spec. No.), S57-S60.

IZUMA, K.; SAITO, D. N.; SADATO, N. (2001): «Processing of social and monetary rewards in the human striatum», Neuron, 58, 284-294.

KENNING, P. H. \& PLASSMANN, H. (2008): «How neuroscience can inform consumer research», IEEE Transactions On Neural Systems And Rehabilitation Engineering, 16, 532-538.

Knutson, B., AdAms, C. M., FONG, G. W.; Hommer, D. (2001): «Anticipation of increasing monetary reward selectively recruits nucleus accumbens», Journal of Neuroscience, 21, RC159.

Knutson, B., Rick, S., Wimmer, G. E., Prelec, D. \& Loewenstein, G. (2007): «Neural predictors of purchases», Neuron, 53, 147-156.

KULYNYCH, J. (2002): «Legal and ethical issues in neu- roimaging research: human subjects protection, medical privacy, and the public communication of research results», Brain and Cognition, 50, 345-357.

ILlES, J; RACINE, E. (2005): «Imaging or imagining? A neuroethics challenge informed by genetics», The American Journal of Bioethics, 5, 5-18. 
LeE, N.; Broderick, A. J.; Chamberlain, L. (2007): «What is neuromarketing? A discussion and agenda for future research», International Journal of Psychophysiology, 63, 199-204.

LeVy, N. (2009): «Neuromarketing: Ethical and Political Challenges», Ethics \& Politics, XI, 2, $10-17$.

Lindstrom, M. (2008): Buyology. Truth and Lies about why we buy, New York, Doubleday. Ed. esp. Buyology. Verdades y mentiras de por qué compramos, Barcelona, Gestión 2000, 2010. MARKETING RESEARCH ASSOCIATION (2007): «The code of marketing research standards». www.mra-net.org, http://www.mra-net.org/images/documents/expanded_code.pdf. Web visitada el 15/02/2011.

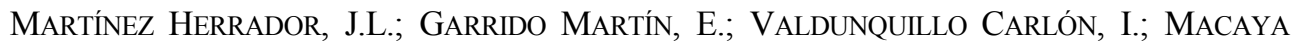
SÁNCHEZ, J. (2010): «Análisis de la atención y la emoción en el discurso político a partir de un nuevo sistema de registro psicofisiológico y su aplicación a las ciencias políticas». Universidad de Salamanca,

http://www.usal.es/ dpublico/Posgrado2009-2010/seminariomartinezherrador.doc. Web consultada el 15/02/2011.

McClure, S. M.; Li, J.; Tomlin, D.; CyPert, K.S.; Montague, L.M.; MonTaGe, R. (2004): «Neural correlates of behavioral preference for culturally familiar drinks», Neuron, 44, 379387.

MuRPHY, E.R,; IlleS, J.; REINER, P.B. (2008): «Neuroethics of neuromarketing», Journal of Consumer Behaviour, 7, 293-302.

NeUROFOCUS (2010): «NeuroFocus Announces NeuroStandardsTM; Market Research Industry's Sole Set of Principles for Conducting Scientifically-Sound, Full Brain-based EEG Studies». Neurofocus.com, http://neurofocus.com/pdfs/NeuroStandardsRelease_NF.PDF. Web visitada el 15/02/2011.

OHMe, R.; ReYKowsKa, D.; WIEner, D.; ChOROMAnsKA, A. (2009): «Analysis of NeuroPhysiological Reactions to Advertising Stimuli by Mean of EEG and Galvanic Skin Response Measures», Journal of Neuroscience, Psychology, and Economics, 2(1), 21-31.

- (2010): «Application of frontal EEG asymmetry to advertising research», Journal of Economic Psychology, 31, 785-793.

PETTY, R.E.; CACIOPPO, J.T. (1986): «The elaboration likelihood model of persuasion». $A d-$ vances in experimental social psychology, 19, 123-205.

PlassmanN, H.; O’DoherTy, J.; Shiv, B.; RANGel, A. (2001): «Marketing actions can modulate neural representations of experienced pleasantness», Proceedings of National Academy of Sciences of the USA, 105, 1050-1054.

Plassmann, H.; KenNing, P.; AhleRT, D. (2007): «Why companies should make their customers happy: The neural correlates of customer loyalty», Advances in Consumer Research - North America Conference Proceedings, 34, 735-739.

REUTERS (2009): «EmSense Raises \$9 Million Series C Round of Financing». Reuters.com, http://www.reuters.com/article/2009/11/03/idUS149271+03-Nov-2009+PRN20091103. Web visitada 21/02/2011.

RoBINSON, J. (2006): «Is That a Neuromarketer in Your Brain?» Gallup Management Journal, January 12. http://gmj.gallup.com/content/20785/neuromarketer-your-brain.aspx. Web visitada el 19/02/2011.

SalaH, A.A.; Sebe, N.; GeVERS, T. (2009): «Communication and automatic interpretation of affect from facial expressions», en Gokay, D. \& Yildirim (eds), Affective Computing and In- 
teraction: Psychological, Cognitive and Neuroscientific perspectives, Hershey (USA), IGI Global, 157-183.

SANDS, S. (2009): «Sample Size Analysis for Brainwave Collection (EEG) Methodologies». Sandsresearch.com, http://www.sandsresearch.com/WhitePaperSampleSize.aspx. Web visitada el 14/02/2011.

Santos, J. P.; SeiXAS, D.; BrandaO, S., MoutinHO, L. (2011): «Investigating the role of the ventromedial prefontral cortex in the assessment of brands», Frontiers in Neuroscience, 5, art. 77, 1-8.

SPIERS, H. J.; MAGUIRE, E. A. (2007): «Decoding human brain activity during real-world experiences», Trends in Cognitive Sciences, 11, 356-365.

TAHERI, B.A.; KNIGHT, R.T.; SMITH, R.L. (1994): «A dry electrode for EEG recording», Electroencephalography and Clinical Neurophysiology, Volume 90, Issue 5, May, 376-383.

THE NIELSEN COMPANY (2008): «Nielsen makes strategic investment in NeuroFocus, an innovative leader in neuromarketing research», Nielsen.com, http://au.nielsen.com/site/NeuroFocus.shtml. Web visitada el 14/02/2011.

TOVINO, SA. (2005): «The confidentiality and privacy implications of functional magnetic resonance imaging», The Journal of Law, Medicine \& Ethics, 33, 844-850.

- (2007): «Functional neuroimaging and the law: trends and directions for future scholarship», The American Journal of Bioethics, 7, 44-56.

WeisberG, D. S.; KeIL, F.C.; GoOdSTEIN, J.; RAWsOn, E.; GrAY, J.R. (2008): «The Seductive Allure of Neuroscience Explanations», Journal of Cognitive Neuroscience, 20(3), 470-477.

WIKIPEDIA (2011a): Electroencefalografía. Dirección http://es.wikipedia.org/wiki/Electroencefalograf\%C3\%ADa Web visitada el 06/02/2011.

- (2011b): Imagen por Resonancia Magnética Funcional. Dirección http://es.wikipedia.org/wiki/Resonancia funcional. Web visitada el 06/02/2011.

- (2011c): Magnetoencefalografía. Dirección http://es.wikipedia.org/wiki/Magnetoencefalograf\%C3\%ADa. Web visitada el 06/02/2011.

Yoon, C.; Gutchess, A. H.; FeINBERG, F.; POLK, T. A. (2006): «A functional magnetic resonance imaging study of neural dissociations between brand and person judgments», Journal of Consumer Research, 33, 31-40.

Zaltman, G; Coutler, R.H. (1995): «Seeing the voice of the Customer: Metaphor-Based Advertising Research», Journal of Advertising Research, 35(4), July/August 1995.

Zaltman, G. (2003): How Customers Think, Boston (MA), Harvard Business School Press. Ed. esp. Cómo piensan los consumidores, Barcelona, Ediciones Urano, 2004. 